\title{
Sound Wave Diffraction by a Cavity with Partial Lining
}

\author{
Burhan Tiryakioglu*
}

\begin{abstract}
Diffraction of sound wave through a cavity with partial lining is analyzed rigorously. Fourier transform technique and Mode Matching method are used to obtain Wiener-Hopf(WH) equation and solution. The solution of the problem is found for various problem parameters numerically. The effect of the different parameters such as the lining length, cavity depth, etc. on the diffraction are illustrated graphically. The results are compared numerically with an existing study and found to be consistent.
\end{abstract}

Keywords: Cavity; Diffraction; Wiener-Hopf Technique; Fourier Transform; Mode Matching.

AMS Subject Classification (2020): Primary: $47 A 68$; Secondary: 42B10; 78A45.

${ }^{*}$ Corresponding author

\section{Introduction}

Waveguide cavities is a well-known subject in acoustic, physics and electromagnetic. It has attracted the interest of investigators studying in this area [1-4]. Diffraction of sound by open ended cavities is significant topic in noise reduction. Different options for reducing noise have been widely discussed in the literature. The concept of absorbent lining is thought to be most appropriate way to reduce unwanted sound. Rawlins proved that acoustically absorbing lining is an efficient method to reduce the irritated sound [5]. The phenomenon of diffraction with acoustically absorbing lining has also been investigated with and without flow by various researchers [6-9].

Demir et al. studied the sound wave diffraction from the cylindrical cavity with inner lining [10]. In their study, Wiener-Hopf method and Mode Matching technique were applied successfully and some numerical results were obtained graphically. Similar problem has been considered for some engineering applications. Matsui discussed the diffraction phenomena of plane sound waves by the actual microphone system [11]. In the literature, parallel problem which is related to the electromagnetic counterpart was studied by Kuryliak [12, 13].

This work was conceived by generalizing the study of [10]. Here, the case in which cavity is partially lined with acoustically absorbing lining is considered. We intend to investigate diffraction properties of the partial lining and the effects on the diffraction phenomenon. By using the waveguide modes in the cavity, the underlying problem is obtained analytically with the help of sophisticated and suitable method known as Wiener-Hopf technique. Numerical solution is found for different parameters of the problem like cavity depth, lining length, etc. The parameters effects on the diffraction are investigated graphically.

The time dependency is taken to be $e^{-i w t}$ where $w$ is the angular frequency.

\section{Problem Formulation}

The diffraction of sound waves by a cavity with partial lining defined by $\{\rho=a, z \in(-\infty, 0)\}$ with $(\rho, \phi, z)$ denote the usual circular cylindrical coordinates, is considered. The outer surface of the semi-infinite cylinder $\left\{\rho=a_{+}, z \in(-\infty, 0)\right\}$ and some part of the interior surface of the cavity $\left\{\rho=a_{-}, z \in\left(-l_{2},-l_{1}\right)\right\}$ are assumed to be rigid while the other part of the inner surface of the cavity $\left\{\rho=a_{-}, z \in\left(-l_{1}, 0\right)\right\}$ and base of the cavity

Received : 20-09-2019, Accepted : 26-11-2019 
$\left\{z=-l_{2}+0, \rho \in(0, a)\right\}$ are coated by absorbing lining which are characterized by a surface impedances $\beta_{1}$ and $\beta_{2}$ respectively. The incident field and the geometry of the problem are symmetric, this provides the independency of



Figure 1. The physical structure of the problem.

$\phi$ of the acoustic field in everywhere. The scalar potential $u(\rho, z)$ is defined the velocity and pressure by $v=\operatorname{grad} u$ and $p=i \omega \rho_{0} u$, respectively. The field of incident is taken as

$$
u_{i}(z)=e^{-i k z}
$$

here $k=\omega / c$ is the wave number. The total field written as

$$
u^{T}(\rho, z)= \begin{cases}u_{1}(\rho, z)+u_{i}(z), & \rho>a, \quad z \in(-\infty, \infty) \\ u_{2}(\rho, z)+u_{i}(z), & \rho<a, \quad z>0 \\ u_{3}(\rho, z), & \rho<a, \quad-l_{1}<z<0 \\ u_{4}(\rho, z), & \rho<a, \quad-l_{2}<z<-l_{1}\end{cases}
$$

$u_{j}, j=1-4$ satisfy the Helmholtz equation in suitable regions. The unknown fields are determined with the continuity and boundary conditions.

$$
\begin{gathered}
\frac{\partial}{\partial \rho} u_{1}(a, z)=0, z<0 \\
\frac{\partial}{\partial \rho} u_{4}(a, z)=0,-l_{2}<z<-l_{1} \\
\left(i k \beta_{1}-\frac{\partial}{\partial \rho}\right) u_{3}(a, z)=0,-l_{1}<z<0 \\
\left(i k \beta_{2}+\frac{\partial}{\partial z}\right) u_{4}\left(\rho,-l_{2}\right)=0, \rho<a \\
\frac{\partial}{\partial \rho} u_{1}(a, z)-\frac{\partial}{\partial \rho} u_{2}(a, z)=0, z>0 \\
u_{1}(a, z)-u_{2}(a, z)=0, z>0 \\
\frac{\partial}{\partial z} u_{2}(\rho, 0)=\frac{\partial}{\partial z} u_{3}(\rho, 0)+i k, \rho<a \\
u_{2}(\rho, 0)=u_{3}(\rho, 0)-1, \rho<a \\
\frac{\partial}{\partial z} u_{3}\left(\rho,-l_{1}\right)=\frac{\partial}{\partial z} u_{4}\left(\rho,-l_{1}\right), \rho<a \\
u_{3}\left(\rho,-l_{1}\right)=u_{4}\left(\rho,-l_{1}\right), \rho<a
\end{gathered}
$$

\section{WH Equation}

Diffracted fields $u_{j}, j=1-4$ satisfy the wave equation for $z \in \mathbb{R}$

$$
\left[\frac{1}{\rho} \frac{\partial}{\partial \rho}\left(\rho \frac{\partial}{\partial \rho}\right)+\frac{\partial^{2}}{\partial z^{2}}+k^{2}\right] u_{j}(\rho, z)=0, j=1-4 .
$$

Consider the Fourier transform of the wave equation satisfied by $u_{1}(\rho, z)$ in the region $\rho>a$ for $z \in \mathbb{R}$

$$
\left[\frac{1}{\rho} \frac{\partial}{\partial \rho}\left(\rho \frac{\partial}{\partial \rho}\right)+K^{2}\right] F(\rho, \alpha)=0
$$


where

$$
K(\alpha)=\sqrt{k^{2}-\alpha^{2}}
$$

which is stated in the complex $\alpha$-plane (Figure 2) and $F(\rho, \alpha)$ is the Fourier transform of the field $u_{1}(\rho, z)$ defined to be

$$
F^{-}(\rho, \alpha)+F^{+}(\rho, \alpha)=\int_{-\infty}^{\infty} u_{1}(\rho, z) e^{i \alpha z} d z=F(\rho, \alpha)
$$

with

$$
F^{+}(\rho, \alpha)=\int_{0}^{\infty} u_{1}(\rho, z) e^{i \alpha z} d z, F^{-}(\rho, \alpha)=\int_{-\infty}^{0} u_{1}(\rho, z) e^{i \alpha z} d z
$$

Through the analyticity of Fourier integrals, $F^{-}(\rho, \alpha)$ and $F^{+}(\rho, \alpha)$ are analytic functions in the lower half plane $(\Im m \alpha<\Im m k)$ and in the upper half plane $(\Im m \alpha>\Im m(-k))$, respectively. The general solution of (3.2) yields

$$
-\frac{A(\alpha) H_{0}^{(1)}(K \rho)}{K H_{1}^{(1)}(K a)}=F^{+}(\rho, \alpha)+F^{-}(\rho, \alpha)
$$

where $A(\alpha)$ is a unknown coefficient and $H_{0}^{(1)}$ is the Hankel function of the first type.

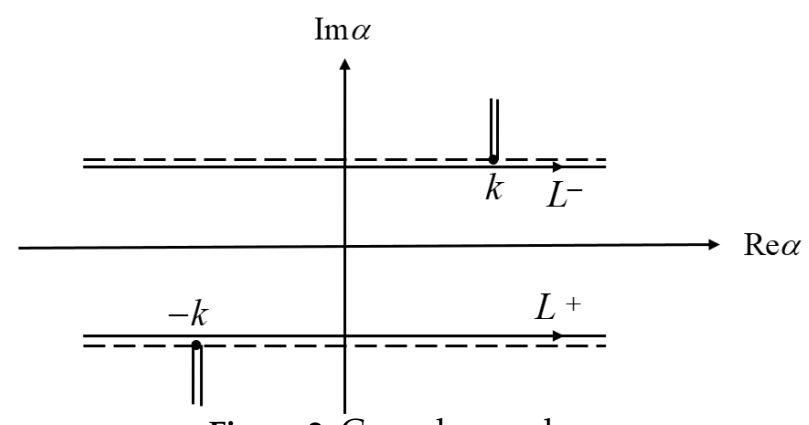

Figure 2. Complex $\alpha$-plane.

The Fourier transform of (2.3), we get

$$
\dot{F}^{-}(a, \alpha)=0
$$

here the $(\cdot)$ defines the derivative according to $\rho$. At $\rho=a$, one can write the following equation by using the derivation of (3.6) with respect to $\rho$

$$
A(\alpha)=\dot{F}^{+}(a, \alpha)
$$

Utilizing (3.8) in (3.6), one obtains

$$
-\frac{\dot{F}^{+}(a, \alpha) H_{0}^{(1)}(K \rho)}{K(\alpha) H_{1}^{(1)}(K a)}=F^{+}(\rho, \alpha)+F^{-}(\rho, \alpha)
$$

For the region $\rho<a, z>0$, the unknown field $u_{2}(\rho, z)$ satisfies the wave equation for $z \in(0, \infty)$ as denoted (3.1). The Fourier transform of this equation for $\rho<a, z>0$ is

$$
\left[\frac{1}{\rho} \frac{\partial}{\partial \rho}\left(\rho \frac{\partial}{\partial \rho}\right)+K^{2}\right] G^{+}(\rho, \alpha)=f(\rho)-i \alpha g(\rho)
$$

The function $G^{+}(\rho, \alpha)$ is an analytic in the upper half plane

$$
G^{+}(\rho, \alpha)=\int_{0}^{\infty} u_{2}(\rho, z) e^{i \alpha z} d z
$$

with

$$
\frac{\partial}{\partial z} u_{2}(\rho, 0)=f(\rho), u_{2}(\rho, 0)=g(\rho)
$$


By applying the Green function, the particular solution of (3.10) can be obtained. The solution is

$$
G^{+}(\rho, \alpha)=-\frac{1}{J_{1}(K a)}\left[B(\alpha) \frac{J_{0}(K \rho)}{K(\alpha)}-\int_{0}^{a}(f(x)-i \alpha g(x)) Q(x, \rho, \alpha) x d x\right]
$$

where

$$
Q(\rho, x, \alpha)=\frac{\pi}{2}\left\{\begin{array}{l}
J_{0}(K \rho)\left[J_{1}(K a) Y_{0}(K x)-Y_{1}(K a) J_{0}(K x)\right], \rho<x \\
J_{0}(K x)\left[J_{1}(K a) Y_{0}(K \rho)-Y_{1}(K a) J_{0}(K \rho)\right], \rho>x
\end{array}\right.
$$

and $B(\alpha)$ is the unknown coefficient to be determined. Taking into account the (2.7), one gets

$$
\dot{G}^{+}(a, \alpha)=B(\alpha)=\dot{F}^{+}(a, \alpha)
$$

Inserting now (3.15) into (3.13) we get

$$
G^{+}(\rho, \alpha)=-\frac{1}{J_{1}(K a)}\left[\dot{F}^{+}(a, \alpha) \frac{J_{0}(K \rho)}{K(\alpha)}-\int_{0}^{a}(f(x)-i \alpha g(x)) Q(x, \rho, \alpha) t d t\right]
$$

Although the left side of (3.16) is regular in the upper half-plane, the right side is not regular. $J_{1}(K a)$ has poles at $\alpha=\alpha_{m}$, satisfying

$$
\begin{gathered}
J_{1}\left(j_{m}\right)=0, m=0,1, \ldots \\
\alpha_{m}=\sqrt{k^{2}-\left(j_{m} / a\right)^{2}}, \alpha_{0}=k, \Im m \alpha_{m} \geq \Im m k
\end{gathered}
$$

To ensure the analyticity of the right side of (3.16), the residue theorem is used. This gives

$$
\dot{F}^{+}\left(a, \alpha_{m}\right)=\frac{a}{2} J_{0}\left(j_{m}\right)\left[f_{m}-i \alpha_{m} g_{m}\right]
$$

with

$$
f_{m}=\frac{2}{a^{2} J_{0}^{2}\left(j_{m}\right)} \int_{0}^{a} f(x) J_{0}\left(\frac{j_{m}}{a} x\right) x d x, g_{m}=\frac{2}{a^{2} J_{0}^{2}\left(j_{m}\right)} \int_{0}^{a} g(x) J_{0}\left(\frac{j_{m}}{a} x\right) x d x
$$

Note that when $m=0, J_{0}\left(j_{0}\right)=1$. Let us expand $f$ and $g$ as [15]

$$
f(\rho)=\sum_{m=0}^{\infty} f_{m} J_{0}\left(\frac{j_{m}}{a} \rho\right), g(\rho)=\sum_{m=0}^{\infty} g_{m} J_{0}\left(\frac{j_{m}}{a} \rho\right)
$$

Now consider the Fourier transform of (2.8) and taking into account (3.16) and (3.9), one gets

$$
\frac{2}{a} \frac{\dot{F}^{+}(a, \alpha)}{K^{2} M(\alpha)}-F^{-}(a, \alpha)=-\frac{1}{a K(\alpha) J_{1}(K a)} \int_{0}^{a}(f(x)-i \alpha g(x)) J_{0}(K x) x d x
$$

with

$$
M(\alpha)=\pi i J_{1}(K a) H_{1}^{(1)}(K a)
$$

By evaluating the integral at the right-hand side of (3.22) after inserting (3.21) in (3.22), we obtain the Wiener-Hopf equation as follows

$$
\frac{\dot{F}^{+}(a, \alpha)}{K^{2}(\alpha) M(\alpha)}-\frac{a}{2} F^{-}(a, \alpha)=\frac{a}{2} \sum_{m=0}^{\infty} \frac{J_{0}\left(j_{m}\right)}{\alpha_{m}^{2}-\alpha^{2}}\left[f_{m}-i \alpha g_{m}\right]
$$




\section{Solution of the WH Equation}

By applying the Wiener-Hopf factorization method, the kernel function can be written

$$
M(\alpha)=M_{-}(\alpha) M_{+}(\alpha), M_{-}(\alpha)=M_{+}(-\alpha)
$$

The functions $M_{-}(\alpha)$ and $M_{+}(\alpha)$ are analytic and free of zeros in the lower and upper half plane, respectively. The term of $M_{+}(\alpha)$ is given in [10] as

$$
\begin{aligned}
M_{+}(\alpha) & =\sqrt{\pi i J_{1}(k a) H_{1}^{(1)}(k a)} \exp \left\{i \frac{a \alpha}{\pi}\left[1-\zeta+\log \left(\frac{2 \pi}{k a}\right)+i \frac{\pi}{2}\right]-\frac{i k a}{2}\right\} \\
& \times \exp \left(\frac{a K(\alpha)}{\pi} \log \left(\frac{\alpha+i K(\alpha)}{k}\right)+q(\alpha)\right) \prod_{m=1}^{\infty}\left(1+\frac{\alpha}{\alpha_{m}}\right) \exp \left(\frac{i \alpha a}{m \pi}\right)
\end{aligned}
$$

where

$$
q(\alpha)=\frac{1}{\pi} \int_{0}^{\infty}\left[1-\frac{2}{\pi x} \frac{1}{J_{1}^{2}(x)+Y_{1}^{2}(x)}\right] \log \left(1+\frac{\alpha a}{\sqrt{(k a)^{2}-x^{2}}}\right) d x
$$

with constant $\zeta=0.57721 \ldots$ The multiplication of both sides of (3.24) by $(k-\alpha) M_{-}(\alpha)$, we get

$$
\frac{\dot{F}^{+}(a, \alpha)}{M_{+}(\alpha)(k+\alpha)}-\frac{a}{2}(k-\alpha) M_{-}(\alpha) F^{-}(a, \alpha)=\frac{a}{2} M_{-}(\alpha)(k-\alpha) \sum_{m=0}^{\infty} \frac{J_{0}\left(j_{m}\right)}{\alpha_{m}^{2}-\alpha^{2}}\left[f_{m}-i \alpha g_{m}\right]
$$

The solution of (4.4) can be obtained by using the Liouville theorem after the decomposition of the right side of (4.4)

$$
\frac{\dot{F}^{+}(a, \alpha)}{(k+\alpha) M_{+}(\alpha)}=\frac{a}{2} \sum_{m=0}^{\infty} \frac{\left(k+\alpha_{m}\right) J_{0}\left(j_{m}\right) M_{+}\left(\alpha_{m}\right)}{2 \alpha_{m}\left(\alpha+\alpha_{m}\right)}\left[f_{m}+i \alpha_{m} g_{m}\right]
$$

\section{Determination of the Expansion Coefficients}

The region for $\rho<a,-l_{1}<z<0$, the field can be defined as

$$
u_{3}(\rho, z)=\sum_{n=1}^{\infty}\left[a_{n} e^{i \kappa_{n} z}+b_{n} e^{-i \kappa_{n} z}\right] J_{0}\left(\frac{\xi_{n}}{a} \rho\right)
$$

Here $\xi_{n}$ 's are the roots of the characteristic equation

$$
i k a \beta_{1} J_{0}\left(\xi_{n}\right)+\xi_{n} J_{1}\left(\xi_{n}\right)=0, n=1,2, \ldots
$$

while $\kappa_{n}$ 's stand for

$$
\kappa_{n}=\sqrt{k^{2}-\left(\frac{\xi_{n}}{a}\right)^{2}}, n=1,2, \ldots
$$

Consider the equations (2.9), (2.10), namely

$$
\begin{aligned}
& f(\rho)=\frac{\partial}{\partial z} u_{2}(\rho, 0)=\frac{\partial}{\partial z} u_{3}(\rho, 0)+i k \\
& g(\rho)=u_{2}(\rho, 0)=u_{3}(\rho, 0)-1
\end{aligned}
$$

Inserting the $f(\rho)$ and $g(\rho)$ given in (3.21) in (5.4), (5.5) respectively, and using (5.1), we get:

$$
\begin{gathered}
\sum_{m=0}^{\infty} f_{m} J_{0}\left(\frac{j_{m}}{a} \rho\right)=i \sum_{n=1}^{\infty} \kappa_{n}\left[a_{n}-b_{n}\right] J_{0}\left(\frac{\xi_{n}}{a} \rho\right)+i k \\
\sum_{m=0}^{\infty} g_{m} J_{0}\left(\frac{j_{m}}{a} \rho\right)=\sum_{n=1}^{\infty}\left[a_{n}+b_{n}\right] J_{0}\left(\frac{\xi_{n}}{a} \rho\right)-1
\end{gathered}
$$


Multiplying both sides of (5.6), (5.7) by $\rho J_{0}\left(\frac{\xi_{l}}{a} \rho\right)$ and integrating from $\rho=0$ to $\rho=a$, we obtain

$$
\begin{aligned}
& a_{n}=\frac{a^{2} \xi_{n} J_{1}\left(\xi_{n}\right)}{2 i \kappa_{n} P_{n}}\left[\sum_{m=0}^{\infty} \frac{J_{0}\left(j_{m}\right)}{\xi_{n}^{2}-j_{m}^{2}}\left[f_{m}+i \kappa_{n} g_{m}\right]+\frac{i\left(\kappa_{n}-k\right)}{\xi_{n}^{2}}\right] \\
& b_{n}=-\frac{a^{2} \xi_{n} J_{1}\left(\xi_{n}\right)}{2 i \kappa_{n} P_{n}}\left[\sum_{m=0}^{\infty} \frac{J_{0}\left(j_{m}\right)}{\xi_{n}^{2}-j_{m}^{2}}\left[f_{m}-i \kappa_{n} g_{m}\right]-\frac{i\left(\kappa_{n}+k\right)}{\xi_{n}^{2}}\right]
\end{aligned}
$$

with

$$
P_{n}=\frac{a^{2}}{2}\left[J_{0}^{2}\left(\xi_{n}\right)+J_{1}^{2}\left(\xi_{n}\right)\right]
$$

In region $\rho<a,-l_{2}<z<-l_{1}, u_{4}(\rho, z)$ can be expressed

$$
u_{4}(\rho, z)=\sum_{n=0}^{\infty}\left[c_{n} e^{-i \alpha_{n} z}+d_{n} e^{i \alpha_{n} z}\right] J_{0}\left(\frac{j_{n}}{a} \rho\right)
$$

By using the (2.6), (5.11) reduces to

$$
u_{4}(\rho, z)=\sum_{n=0}^{\infty} c_{n}\left[e^{-i \alpha_{n} z}+R_{n} e^{i \alpha_{n} z}\right] J_{0}\left(\frac{j_{n}}{a} \rho\right)
$$

with

$$
R_{n}=-\frac{\beta_{2}-\alpha_{n} / k}{\beta_{2}+\alpha_{n} / k} e^{2 i \alpha_{n} l_{2}}
$$

Now from (2.11), (2.12) and using (5.1), (5.12) we write

$$
\begin{aligned}
\sum_{n=1}^{\infty} \kappa_{n}\left[a_{n} e^{-i \kappa_{n} l_{1}}-b_{n} e^{i \kappa_{n} l_{1}}\right] J_{0}\left(\frac{\xi_{n}}{a} \rho\right) & =\sum_{m=0}^{\infty} c_{m} \alpha_{m}\left[-e^{i \alpha_{m} l_{1}}+R_{m} e^{-i \alpha_{m} l_{1}}\right] J_{0}\left(\frac{j_{m}}{a} \rho\right) \\
\sum_{n=1}^{\infty}\left[a_{n} e^{-i \kappa_{n} l_{1}}+b_{n} e^{i \kappa_{n} l_{1}}\right] J_{0}\left(\frac{\xi_{n}}{a} \rho\right) & =\sum_{m=0}^{\infty} c_{m}\left[e^{i \alpha_{m} l_{1}}+R_{m} e^{-i \alpha_{m} l_{1}}\right] J_{0}\left(\frac{j_{m}}{a} \rho\right)
\end{aligned}
$$

Similarly one can obtain

$$
\begin{gathered}
a_{n}=\frac{e^{i \kappa_{n} l_{1}} a^{2} \xi_{n} J_{1}\left(\xi_{n}\right)}{2 \kappa_{n} P_{n}} \sum_{m=0}^{\infty} \frac{c_{m}\left[\alpha_{m}\left[-e^{i \alpha_{m} l_{1}}+R_{m} e^{-i \alpha_{m} l_{1}}\right]+\kappa_{n}\left[e^{i \alpha_{m} l_{1}}+R_{m} e^{-i \alpha_{m} l_{1}}\right]\right]}{\xi_{n}^{2}-j_{m}^{2}} J_{0}\left(j_{m}\right) \\
b_{n}=\frac{e^{-i \kappa_{n} l_{1}} a^{2} \xi_{n} J_{1}\left(\xi_{n}\right)}{2 \kappa_{n} P_{n}} \sum_{m=0}^{\infty} \frac{c_{m}\left[-\alpha_{m}\left[-e^{i \alpha_{m} l_{1}}+R_{m} e^{-i \alpha_{m} l_{1}}\right]+\kappa_{n}\left[e^{i \alpha_{m} l_{1}}+R_{m} e^{-i \alpha_{m} l_{1}}\right]\right]}{\xi_{n}^{2}-j_{m}^{2}} J_{0}\left(j_{m}\right)
\end{gathered}
$$

consider (5.8), (5.9) together with (5.16), (5.17), namely

$$
\begin{aligned}
\sum_{m=0}^{\infty} \frac{J_{0}\left(j_{m}\right)}{\xi_{n}^{2}-j_{m}^{2}}\left[f_{m}\right. & \left.+i \kappa_{n} g_{m}\right] \\
=i e^{i \kappa_{n} l_{1}} \sum_{m=0}^{\infty} \frac{c_{m}\left[\alpha_{m}\left[-e^{i \alpha_{m} l_{1}}+R_{m} e^{-i \alpha_{m} l_{1}}\right]+\kappa_{n}\left[e^{i \alpha_{m} l_{1}}+R_{m} e^{-i \alpha_{m} l_{1}}\right]\right]}{\xi_{n}^{2}-j_{m}^{2}} J_{0}\left(j_{m}\right) & -\frac{i\left(\kappa_{n}-k\right)}{\xi_{n}^{2}}
\end{aligned}
$$




$$
\begin{aligned}
\sum_{m=0}^{\infty} \frac{J_{0}\left(j_{m}\right)}{\xi_{n}^{2}-j_{m}^{2}}\left[f_{m}-i \kappa_{n} g_{m}\right] & \\
=-i e^{-i \kappa_{n} l_{1}} \sum_{m=0}^{\infty} \frac{c_{m}\left[-\alpha_{m}\left[-e^{i \alpha_{m} l_{1}}+R_{m} e^{-i \alpha_{m} l_{1}}\right]+\kappa_{n}\left[e^{i \alpha_{m} l_{1}}+R_{m} e^{-i \alpha_{m} l_{1}}\right]\right]}{\xi_{n}^{2}-j_{m}^{2}} J_{0}\left(j_{m}\right) & +\frac{i\left(\kappa_{n}+k\right)}{\xi_{n}^{2}}
\end{aligned}
$$

By replacing $\alpha=\alpha_{1}, \alpha_{2}, \alpha_{3}, .$. in (4.5) and using (3.19), we get

$$
\frac{J_{0}\left(j_{r}\right)\left[f_{r}-i \alpha_{r} g_{r}\right]}{\left(k+\alpha_{r}\right) M^{+}\left(\alpha_{r}\right)}=\sum_{m=0}^{\infty} \frac{\left(k+\alpha_{m}\right) M^{+}\left(\alpha_{m}\right) J_{0}\left(j_{m}\right)}{2 \alpha_{m}\left(\alpha_{r}+\alpha_{m}\right)}\left[f_{m}+i \alpha_{m} g_{m}\right]
$$

One can determines the coefficients $f_{m}, g_{m}$ and $c_{m}$ by using the linear systems (5.18), (5.19) and (5.20).

\section{Far Field}

The diffracted field for $\rho>a$ can be found by applying the inverse Fourier transform of $F(\rho, \alpha)$. From (11b) we get

$$
u_{1}(\rho, z)=-\frac{1}{2 \pi} \int_{\mathcal{L}} \frac{\dot{F}^{+}(a, \alpha) H_{0}^{(1)}(K \rho)}{K H_{1}^{(1)}(K a)} e^{-i \alpha z} d \alpha
$$

Here $\mathcal{L}$ is a line parallel to the real $\alpha$-axis. By using the asymptotic expression of $H_{0}^{(1)}(K \rho)$ for $k \rho \rightarrow \infty$

$$
H_{0}^{(1)}(K \rho)=\sqrt{\frac{2}{\pi K \rho}} e^{i K \rho-i \pi / 4}
$$

The substitution $\alpha=-k \cos \mu$ in (6.1) enables us to write

$$
u_{1}(\rho, z)=-\frac{1}{2 \pi} \int_{\mathcal{L}} \frac{\dot{F}^{+}(a,-k \cos \mu)}{k \sin \mu H_{1}^{(1)}(k a \sin \mu)} \sqrt{\frac{2}{\pi \rho k \sin \mu}} e^{i \rho k \sin \mu+i k z \cos \mu-i \pi / 4} k \sin \mu d \mu
$$

and making the following substitutions

$$
z=r \cos \theta, \rho=r \sin \theta
$$

in (6.3), the integral can be solved owing to the saddle-point formula [16], we have

$$
u_{1}(r, \theta) \sim \frac{i}{\pi} \frac{\dot{F}^{+}(a,-k \cos \theta)}{\sin \theta H_{1}^{(1)}(k a \sin \theta)} \frac{e^{i k r}}{k r}
$$

where $\theta$ and $r$ are the spherical coordinates.

\section{Numerical Results}

In order to show the influence of the parameters on the diffracted field phenomenon, numerical results are presented. Graphics are obtained for $20 \log \left|u_{1}(r, \theta) \times k r\right|$ with the observation angle $\theta$ changing from 0 to $\pi$.

Figure 3 shows that the modulus of the diffracted field becomes insensitive to the increasing value of $N>5$ when $k a=1, k l_{1}=1, k l_{2}=10, \beta_{1}^{-1}=1+3 i, \beta_{2}^{-1}=3+3 i$. For this reason, in all numerical computations $N$ is chosen as 5 .

Figures 4 and 5 show the variation of the diffracted field amplitude versus observation angle $\theta$ for different values of $k l_{1}$. The diffracted field amplitude decreases with increasing lining length, as expected. In Figure 5, the effect obtained by coating the cavity is also achieved by partial lining of the cavity, depending on the selection of the problem parameters.

Figure 6 depicts the effect of the cavity depth $k l_{2}$ on the diffraction phenomenon. The diffracted field becomes almost constant (no resonance effect) after $k=5$ due to the complex impedances, is observed.

Comparison of the amplitudes of diffracted field and cavity depth are presented in Figures 7 and 8 . These Figures show an excellent agreement between the present study $\left(k l_{1} \rightarrow k l_{2}\right)$ and the study of [10]. This comparison is also important for the accuracy of the mathematical results. 




Figure 3. Diffracted field against the truncation number with different observation angle.

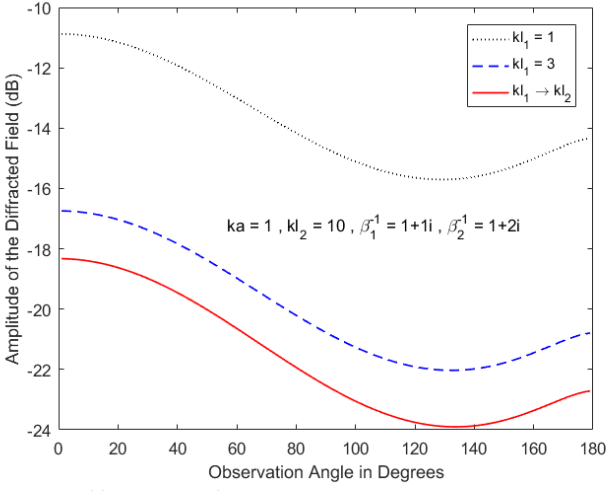

Figure 4. Diffracted field against the cavity depth $k l_{1}$.

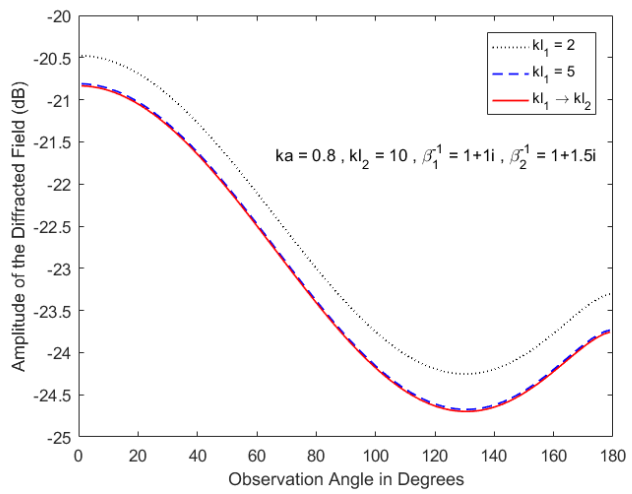

Figure 5. Diffracted field versus cavity depth $k l_{1}$. 


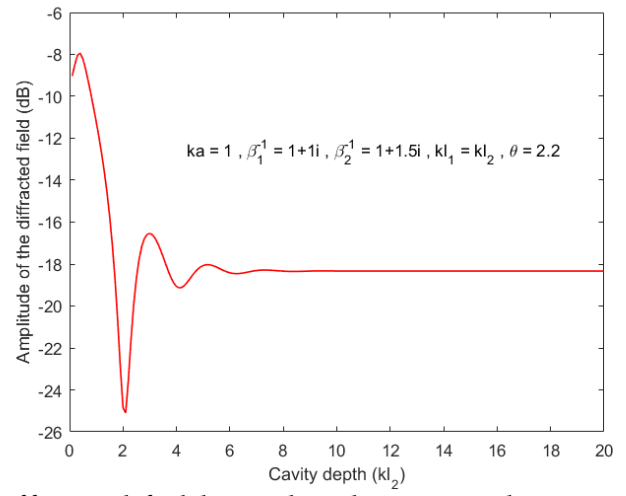

Figure 6. Diffracted field amplitude versus the cavity depth $k l_{2}$.

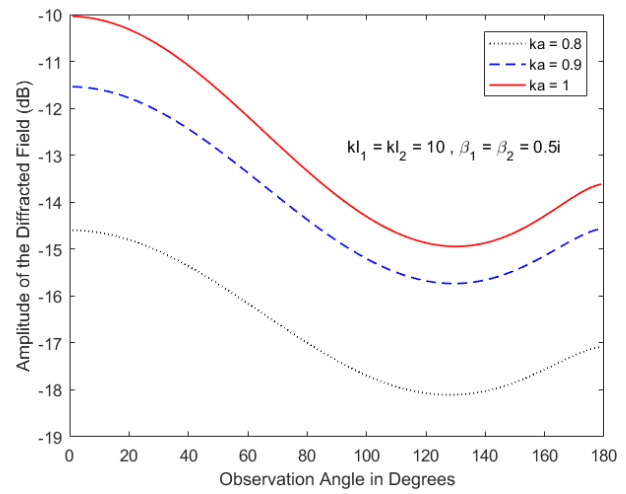

Figure 7. Comparison of the diffracted field with the study of [10].



Figure 8. Comparison of the cavity depth with the study of [10]. 


\section{Conclusions}

In this present study, diffraction of sound waves from cavity with partial lining is presented rigorously. By using the Fourier transform, boundary and continuity condition, the problem reduced to WH equation. The analytic solution is obtained by using the WH equation. At the end of the analysis, the inverse Fourier transform is applied to determine the explicit expressions of the diffracted field. Also, some numerical results are presented to show the effects of the some physical parameters to the diffracted field.

As is well known, the lining of the cavity provided a few decibel of sound wave reduction. In addition, the effect of partial lining on diffracted field is clearly seen from Figures 4,5. Considering the cost and applicability, the importance of a finite coating is obvious.

When the cavity is full lined $\left(k l_{1} \rightarrow k l_{2}\right)$, the results found in this study are compared with the results of [10] and the agreement is perfect.

\section{References}

[1] Kobayashi, K., Sawai, A.: Plane Wave Diffraction by an Open-Ended Parallel Plate Waveguide Cavity. Journal of Electromagnetic Waves and Applications. 6, 475-512 (1992).

[2] Victoria, A., Kimberly, S.R., Jonathan, J., Rajind, M., Daniel, M.M.: Terahertz multichannel microfluidic sensor based on parallel-plate waveguide resonant cavities. Applied Physics Letters. 100, 231108 (2012).

[3] Tong, Y., Pan, J.: Modal analysis of the scattering coefficients of an open cavity in a waveguide. Wave Motion. 68, 242-252 (2017).

[4] Porter, R., Evans, D.V.: Analysis of the effect of a rectangular cavity resonator on acoustic wave transmission in a waveguide. Journal of Sound and Vibration. 408, 138-153 (2017).

[5] Rawlins, A.D.: Radiation of sound from an unflanged rigid cylindrical duct with an acoustically absorbing internal surface. Proceedings of the Royal Society A: Mathematical, Physical and Engineering Sciences. 361, 65-91 (1978).

[6] Rienstra, S.W.: Acoustic scattering at a hard-soft lining transition in a flow duct. Journal of Engineering Mathematics. 59, 451-475 (2007).

[7] Snakowska, A., Jurkiewicz, J., Gorazd, L.: A hybrid method for determination of the acoustic impedance of an unflanged cylindrical duct for multimode wave. Journal of Sound and Vibration. 396, 325-339 (2017).

[8] Tiryakioglu, B., Demir, A.: Radiation analysis of sound waves from semi-infinite coated pipe. International Journal of Aeroacoustics. 18 (1), 92-111 (2019).

[9] Tiryakioglu B., Demir, A.: Sound Wave Radiation from Partially Lined Duct. Archives of Acoustics. 44 (2), 239-249 (2019).

[10] Demir, A., Buyukaksoy, A., Polat, B.: Diffraction of plane sound wave by a rigid circular cylindrical cavity with an acoustically absorbing internal surface. Journal of Applied Mathematics and Mechanics. 82 (9), 619-629 (2002).

[11] Matsui, E.: Free-Field Correction for Laboratory Standard Microphones Mounted on a Semiinfinite Rod. The Journal of the Acoustical Society of America. 49, 1475-1483 (1971).

[12] Kuryliak, D.B., Koshikawa, S., Kobayashi, K., Nazarchuk, Z.T.: Wiener-Hopf analysis of the vector diffraction problem for a cylindrical waveguide cavity. In: Conference Proceedings 2000 International Conference on Mathematical Methods in Electromagnetic Theory, Sept 12-15 2000, Kharkov, Ukraine.

[13] Kuryliak, D.B, Koshikawa, S., Kobayashi, K., Nazarchuk, Z.T.: Wiener-Hopf analysis of the axial symmetric wave diffraction problem for a circular waveguide cavity. In: Proceeding of the International Workshop on Direct and Inverse Wave Scattering, Sept 25-29 2000, Gebze, Turkey.

[14] Noble, B.: Methods Based on the Wiener-Hopf Technique. Chelsea Publishing Company, New York 1988.

[15] Watson, G.N.: A Treatise on the Theory of Bessel Functions. Cambridge University Press, London 1944.

[16] Mittra, R., Lee S.W.: Analytical Techniques in the Theory of Guided Waves. The Macmillan Company, New York 1971. 


\section{Affiliation}

BURHAN TIRYAKIOGLU

ADDRESS: Marmara University, Department of Applied Mathematics, 34722, Istanbul-Turkey. E-MAIL: burhan.tiryakioglu@marmara.edu.tr

ORCID ID: 0000-0003-1448-6147 